\title{
Accounting for Anisotropic Noise in Fine Registration of Time-of-Flight Range Data with High-Resolution Surface Data
}

\author{
L. Maier-Hein ${ }^{1}$, M. Schmidt ${ }^{2}$, A.M. Franz ${ }^{1}$, T.R. dos $\operatorname{Santos}^{1}$, A. Seitel ${ }^{1}$, \\ B. Jähne ${ }^{2}$, J.M. Fitzpatrick ${ }^{3, \star}$, and H.P. Meinzer ${ }^{1}$ \\ 1 Div. Medical and Biological Informatics, German Cancer Research Center \\ 1.maier-hein@dkfz-heidelberg.de \\ 2 Heidelberg Collaboratory for Image Processing, University of Heidelberg, Germany \\ 3 Dept. Electrical Engineering and Computer Science, Vanderbilt University, USA
}

\begin{abstract}
Time-of-Flight (ToF) sensors have become a considerable alternative to conventional surface acquisition techniques such as laser range scanning and stereo vision. Application of ToF cameras for the purpose of intra-operative registration requires matching of the noisy surfaces generated from ToF range data onto pre-interventionally acquired high-resolution surfaces. The contribution of this paper is twofold: Firstly, we present a novel method for fine rigid registration of noisy ToF data with high-resolution surface meshes taking into account both, the noise characteristics of ToF cameras and the resolution of the target mesh. Secondly, we introduce an evaluation framework for assessing the performance of ToF registration methods based on physically realistic ToF range data generated from a virtual scence. According to experiments within the presented evaluation framework, the proposed method outperforms the standard ICP algorithm with respect to correspondence search and transformation computation, leading to a decrease in the target registration error (TRE) of more than $70 \%$.
\end{abstract}

\section{Introduction}

A growing number of applications in the field of computer-assisted medical interventions depend on accurate and fast 3D surface acquisition. To date, however, the estimation of a range map by image analysis or other techniques is still a challenging and time-consuming task. A novel fast and robust alternative for distance measurements are Time-of-Flight (ToF) cameras, which provide range images in addition to gray-scale intensity images with high update rates [2]. Processing ToF data typically involves registration of the acquired distance images with pre-operative volume data.

The Iterative Closest Point (ICP) algorithm [3] is a widely used method for geometric alignment of 3D models. Given two roughly aligned shapes represented by two point sets, it iteratively (1) establishes point correspondences given the

\footnotetext{
* Thanks to R. Balachandran for the source code of the algorithm described in 1 .
} 


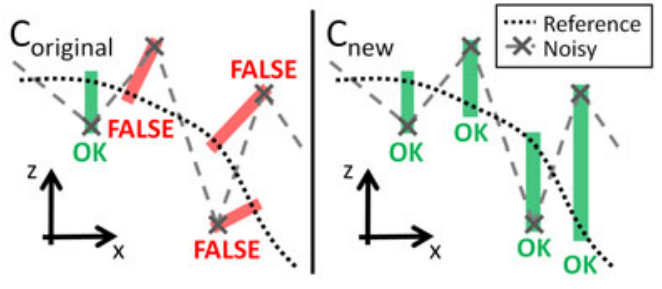

(a)

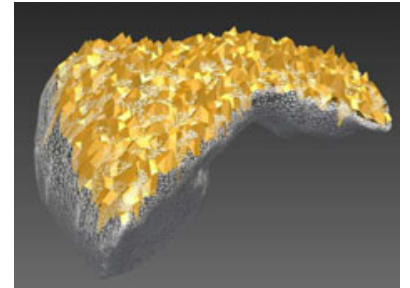

(b)

Fig. 1. (a) Schematic illustration of the establishment of point correspondences with the original ICP (left) and the anisotropic ICP (right). Reference mesh (represented by dotted black line), sparse noisy mesh (represented by gray crosses) and correspondences (boxes) are shown for $C_{\text {original }}$ (standard closest point operator) and $C_{\text {new }}$ (new closest point operator with less weight given to the direction $z$. (b) Noisy submesh registered to a reference liver mesh via the proposed registration method.

current alignment of the data and (2) computes a rigid transformation accordingly. Although widely used, the algorithm implicitly assumes that the input points are observed with zero-mean, identical and isotropic Gaussian noise. The statistical errors related to the process of generating $3 \mathrm{D}$ points from ToF range data, however, lead to highly anisotropic error distributions in the point data. This, in turn, may result in wrong assignments during a correspondence search, as illustrated in Fig. 1(a).

In a related paper, we proposed a new anisotropic variant of the ICP [4] which allows for definition of a covariance matrix for each point in the noisy input set. In this paper, we extend the algorithm, such that a covariance matrix can be defined for each point in both point sets to be aligned and show how to apply the derived variant of the ICP for fine registration of noisy ToF images with high-resolution surface data (sec. 2.1). The performance of the proposed method is assessed with a novel evaluation framework that provides physically realistic ToF range data generated from a virtual scence (sec. 2.2).

\section{Materials and Methods}

\subsection{Method for Fine Registration of ToF Range Data}

Let $X=\left\{\boldsymbol{x}_{1}, \ldots, \boldsymbol{x}_{N_{\boldsymbol{X}}}\right\}$ be a sparse point set acquired with a ToF camera to be registered with a dense reference set $Y=\left\{\boldsymbol{y}_{1}, \ldots, \boldsymbol{y}_{N_{Y}}\right\}$ (e.g., the set of vertices from a surface mesh). Given two points $\boldsymbol{x}$ and $\boldsymbol{y}$, whose localization errors are assumed to be independent and can be represented by two zero-mean Gaussian distributions with covariance matrices $\Sigma_{\boldsymbol{x}}$ and $\Sigma_{\boldsymbol{y}}$ (rank 3), we define the anisotropically weighted distance between them as:

$$
d_{n e w}(\boldsymbol{x}, \boldsymbol{y})=\left\|W_{\boldsymbol{x} \boldsymbol{y}}(\boldsymbol{x}-\boldsymbol{y})\right\|_{2}
$$


where a weighting matrix $W_{\boldsymbol{x} \boldsymbol{y}}=\left(\Sigma_{\boldsymbol{x}}+\Sigma_{\boldsymbol{y}}\right)^{-\frac{1}{2}}$ accounts for the variance in the input data similar as in 1445 . The closest point in a set $Y$ to a given point $\boldsymbol{x}$ is then defined as:

$$
C_{n e w}(\boldsymbol{x}, Y)=\arg \min _{\boldsymbol{y}_{\boldsymbol{i}} \in Y}\left\|W_{\boldsymbol{x} \boldsymbol{y}_{i}}\left(\boldsymbol{x}-\boldsymbol{y}_{i}\right)\right\|_{2}
$$

Based on the provided covariance matrices $\Sigma_{\boldsymbol{p}}$ for each point $\boldsymbol{p} \in X \cup Y$, the aim of the anisotropic ICP algorithm is to find a rotation matrix $R$ and a translation vector $\boldsymbol{t}$ such that the following error metric is minimized:

$$
e(R, \boldsymbol{t})=\frac{1}{N_{X}} \sum_{i=1}^{N_{X}}\left\|W_{i}\left(R \boldsymbol{y}_{i d x(i)}+\boldsymbol{t}-\boldsymbol{x}_{i}\right)\right\|_{2}^{2}
$$

where $i d x(i)=\operatorname{argmin}{ }_{j} d_{n e w}\left(\boldsymbol{x}_{i}, \tilde{\boldsymbol{y}}_{j}\right)$ with $\tilde{\boldsymbol{y}}_{j}=R \boldsymbol{y}_{j}+\boldsymbol{t}, j=1, \ldots, N_{Y}$ and $W_{i}=\left(R \Sigma_{\boldsymbol{y}_{i d x(i)}} R^{\prime}+\Sigma_{\boldsymbol{x}_{i}}\right)^{-\frac{1}{2}}$.

Similar as in 4, this is achieved by iteratively (1) establishing point correspondences with the new closest point operator $C_{n e w}$ given the current alignment of the data and (2) computing a rigid transformation for mapping the current corresponding points using an extension of the recently published algorithm by Balachandran and Fitzpatrick 1]. The latter minimizes an anisotropically weighted FRE, where the weighting matrix for two corresponding points is a function of their covariance matrices as depicted above. It can be shown, that the cost function given in eq. 3 decreases in each iteration of the proposed anisotropic ICP (proof by contradiction).

To apply the anisotropic ICP for registration of two surfaces represented by the point sets $X$ and $Y$, it is necessary to define a covariance matrix $\Sigma_{p}$ for each $\boldsymbol{p} \in X \cup Y$ representing the localization uncertainty in that point. We propose the following three variants for defining $\Sigma_{\boldsymbol{x}}$ for a point $\boldsymbol{x} \in X$ on the ToF surface:

ANISOTROPIC: We assume that the localization error for each point occurs primarily along the ray connecting the imaged object point $\boldsymbol{x}$ with the center of the associated chip pixel and set the remaining two principal axes orthogonal to that ray. Let $V_{\boldsymbol{x}}$ be the matrix whose columns represent the normalized principal axes. We then set $\Sigma_{\boldsymbol{x}}=V_{\boldsymbol{x}} S_{\boldsymbol{x}}^{2} V_{\boldsymbol{x}}^{\prime}$ with $S_{\boldsymbol{x}}=\operatorname{diag}\left(s_{\boldsymbol{x} \mathbf{1}}, s_{\boldsymbol{x} \mathbf{2}}, s_{\boldsymbol{x} \mathbf{3}}\right)$. The standard deviation $s_{\boldsymbol{x} 1}$ along the ray is set to a constant value depending on the distance of the examined object to the camera (default: $3 \mathrm{~mm}$ ), whereas $s_{\boldsymbol{x} 2}$ and $s_{\boldsymbol{x} \mathbf{3}}$ represent the lateral statistical error and are set to the empirically determined value $s_{\boldsymbol{x} \mathbf{2}}=s_{\boldsymbol{x} \mathbf{3}}=0.1 \mathrm{~mm}$. Note that in this variant, the covariance matrices can be computed prior to image acquisition.

ANISOTROPIC_SIM: A variant of ANISOTROPIC where the variance along the first principal axis is determined with a ToF simulator: For a given illumination power, reflectivity of the imaged object, as well as optics characteristics, the standard deviation can be determined as a function of the measured distance $d(\boldsymbol{x})$ of $\boldsymbol{x}$ to the camera: $s_{\boldsymbol{x} \mathbf{1}}=f(d(\boldsymbol{x}))$. This function can be determined by computing $f\left(d_{i}\right)$ for a set of distances $d_{i}$ using the simulator presented in sec. 2.2 
and fitting a spline to the output data. The performed simulations can be seen as a training for the method.

ANISOTROPIC_EST: A variant of ANISOTROPIC where the variance along the first principal axis is estimated from the depth values corresponding to $\boldsymbol{x}$ from the last $n$ samples (default: $n=10$ ). Note that the standard deviation needs to be computed from a relatively small amount of data to avoid motion artefacts.

When registering the mean image of a set of $n$ ToF images, the standard deviations computed for a single image are divided by $\sqrt{n}$.

To account for the resolution of the mesh represented by $Y$, we set the first two principal axes $\boldsymbol{v}_{\boldsymbol{y} \mathbf{1}}, \boldsymbol{v}_{\boldsymbol{y} \mathbf{2}}$ for a point $\boldsymbol{y} \in Y$ in that mesh orthogonal to the normal vector $\boldsymbol{n}$ of the corresponding vertex. The standard deviation along these axes is set to the radius of a circle with area $A_{V}(\boldsymbol{y})$, where $A_{V}(\boldsymbol{y})$ is the area of the Voronoi region corresponding to $\boldsymbol{y}$. The rationale behind this procedure is that the variance along the surface should increase with an increasing size of the triangles associated with this vertex to ensure that corresponding vertices in two meshes to be registered do not need to correspond to exactly the same anatomical location. Note, however, that different spreads along different directions are not accounted for. The standard deviation $s_{\boldsymbol{y}}$ along the vertex normal can either be set to a value close to 0 , thus representing a noise-free target mesh, or to a value inversely proportional to the intensity gradient in that point, thus reflecting the segmentation error (provided that the mesh was generated from volume data). We then set $\Sigma_{\boldsymbol{y}}=V_{\boldsymbol{y}} S_{\boldsymbol{y}}^{2} V_{\boldsymbol{y}}^{\prime}$ with $S_{\boldsymbol{y}}=\operatorname{diag}\left(s_{\boldsymbol{y} \mathbf{1}}, s_{\boldsymbol{y} \mathbf{2}}, s_{\boldsymbol{y} \mathbf{3}}\right)$ and $V_{\boldsymbol{y}}=\left[\begin{array}{ll}\boldsymbol{v}_{\boldsymbol{y} \mathbf{1}} & \boldsymbol{v}_{\boldsymbol{y} \mathbf{2}} \boldsymbol{n}\end{array}\right]$.

\subsection{Evaluation Framework}

The evaluation framework consists of the following components (cf. Fig. 2):

ToF Surface Generator: This component generates a noisy ToF mesh from a given high-resolution surface mesh based on a chosen camera type (default: CamCube 2.0; PMDTechnologies GmbH, Siegen, Germany), a pose of the camera relative to the object and (optionally) the object's reflectivity (per vertex). It consists of three modules. The ideal depth map generator provides means for placing the input mesh relative to the chosen ToF camera in a virtual scene and generates a corresponding ideal depth map based on the intrinsic camera parameters. In the second step, the ToF camera simulator generates a noisy depth map from the ideal depth map, taking into account realistic sensor properties like the quantum efficiency, dark currents, system amplification factors as well as the digitization process. It applies a previously proposed concept for ToF sensor simulation [6] and additionally simulates the attenuation of illumination intensity with depth as well as the lateral statistical error caused by non-ideal optics. The latter is achieved by convoluting the input of the virtual camera with a Gaussian blur kernel prior to surface generation. Systematic errors, such as pixel offsets and the periodical depth deviation (wiggling error) are not simulated because unlike statistical errors, they can be compensated for by a calibration 


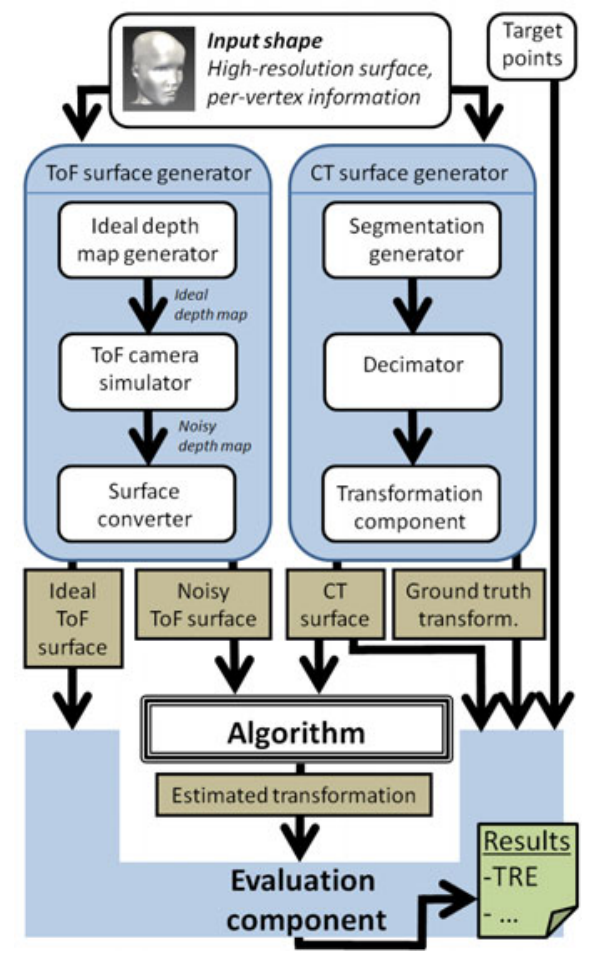

Fig. 2. Evaluation framework developed for this study (cf. sec. 2.2)

procedure based on reference data 2]. Finally, the surface converter generates a surface from the noisy depth map based on the intrinsic camera parameters. All points with a corresponding distance value above a certain threshold are excluded, hence, an object segmentation is automatically generated.

CT Surface Generator: This component generates a surface representing the reference input mesh for the algorithm to be evaluated. Segmentation errors can be simulated by adding noise to the vertices based on the intensity gradient in that point, and a decimator can be applied for modifying the resolution of the mesh. Finally, an initial pose relative to the ToF surface in the world coordinate system can be chosen.

Evaluation Component: Based on a set of provided target points, the output of the other components and the transformation computed by the algorithm to be evaluated, this module computes the target registration error (TRE), which we define as the root-mean-square (RMS) distance between the ground truth target positions and the corresponding target positions according to the transformation computed by the algorithm. Furthermore, the translation error and the rotational error are determined. If the algorithm under evaluation yields vertex 
correspondences (such as the ICP), the percentage of correct vertex correspondences is additionally determined. Ground truth correspondences are calculated by transforming the ideal depth map into a 3D point cloud and determining the closest vertex in the simulated CT mesh for each ToF mesh point.

\section{$2.3 \quad$ Experiments}

Artificial Setting: The goal of the first experiment was to demonstrate with a simple example that the proposed algorithm is better suited for coping with anisotropic noise than the original ICP algorithm. For this purpose, an object consisting of two sides arranged perpendicular to each other (imagine a book opened with an angle of $90^{\circ}$ ) was placed in the world coordinate system such that the direction of view of the virtual ToF camera was almost perpendicular to one of the two sides of the object and almost parallel to the other side (a small portion of which was still visible). The idea behind this was, that - similar as in the example shown in Fig. 1(a) - wrong assignments with the standard ICP should primarily occur in one direction (towards the other side) and could not be compensated for by wrong assignments in the opposite direction. Based on an ideal depth map generated by the ideal depth map generator, a noisy depth map was generated by the ToF camera simulator with a simulated exposure time of $25 \mathrm{~ms}$ and a modulation contrast of the light source of 0.5. All sensor parameters (quantum efficiency, dark currents, etc.) were set to values measured for the CamCube 2.0 ToF system using methods proposed by Erz et al. 7 . The illumination power and aperture of the optics were set to values which led to $90 \%$ pixel saturation on average. The optics influence was simulated as a Gaussian point spread function with a full width at half maximum (FWHM) of $20 \mu \mathrm{m}$. Next, a noisy partial surface was generated with the surface converter, and both, the original ICP and the proposed variant were used to register the noisy surface to the high-resolution surface mesh, starting from the ideal position and assuming a noise-free reference mesh $\left(s_{\boldsymbol{y} \mathbf{3}}=10^{-20}\right)$. For both algorithms, the percentage of correct correspondences as well as the TRE for a set of points distributed in the target object were determined.

Medical Setting: To evaluate the performance of the proposed ICP in the medical context, three high-resolution surface meshes were generated from medical imaging data: a liver with little depth variation (LIVER1), a liver with more depth variation (LIVER2) and a face mesh (FACE). For the livers, the targets were distributed within the organ itself. In the case of the face, the targets were distributed in the corresponding brain segmentation. For each mesh, an ideal ToF image was created with an approximately ventral view of the camera on the patient. These images were averaged to obtain a mean depth map which was then converted into a noisy partial surface using the same parameters as in the Artificial setting. Both, the standard ICP and the proposed variant of the ICP were then used to register the noisy ToF mesh (between 1800 and 3000 points) with the original mesh (approximately 10000 points), starting with a small known initial misalignment. For each image and each method, the TRE and the percentage of correct point correspondences was computed. 


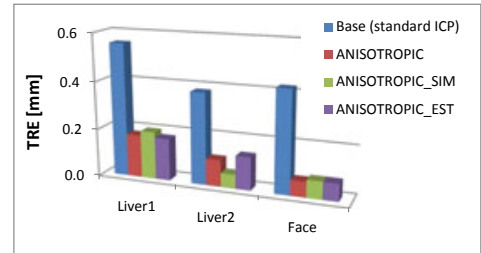

(a) TRE

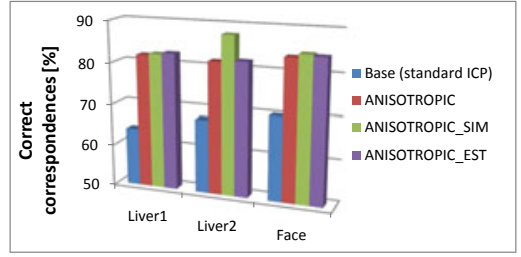

(b) Correspondences

Fig. 3. Summary of the results for the medical setting showing (a) the TRE and (b) the percentage of correct matches after convergence for the different registration methods and algorithms

\section{Results}

Registration of the artificial object with the standard ICP yielded a TRE of $2.9 \mathrm{~mm}$ compared to $0.5 \mathrm{~mm}$ (ANISOTROPIC), $0.4 \mathrm{~mm}$ (ANISOTROPIC_SIM), and $0.4 \mathrm{~mm}$ (ANISOTROPIC_EST) with the proposed algorithm. In the former case (standard ICP), the percentage of correct correspondences was $23.1 \%$ in the ground truth position and $0.8 \%$ after ICP convergence, which could be increased to $(30 \pm 1) \%$ and $(27 \pm 1) \%$ respectively when applying the new registration method (averaged over the three variants). The registration results for the medical imaging data are shown in Fig. 3. Again, the different variants of the proposed algorithm performed similarly, yielding a mean decrease of the TRE of $(73 \pm 2) \%$, and a mean increase of the percentage of correct correspondences of $(24 \pm 2) \%$ on average. A typical registration result is shown in Fig. 1(b).

\section{Discussion}

We introduced a new method for fine registration of partial ToF surfaces with high-resolution surface meshes based on an anisotropic variant of the ICP algorithm and assessed its performance with a novel evaluation framework. To our knowledge, we are the first to present a framework for ToF registration algorithms based on physically realistic ToF sensor simulations. The simulated sources of errors include various errors related to the process of generating a ToF depth image from a given scene such as the influence of interfering nonmodulated background light and dark currents, as well as errors caused in the process of mesh segmentation and mesh decimation. As the framework generates ideal ToF surfaces in addition to the noisy ones, it is also suitable for evaluation of ToF preprocessing algorithms (such as denoising methods).

According to our experiments, the proposed method is better suited for fine registration of ToF range data than the standard ICP, reducing the TRE by over $70 \%$ and increasing the percentage of correct matches by more than $20 \%$ on medical imaging data. Although the percentage of correct matches was always below $70 \%$ for the standard ICP, the absolute value of the TRE was quite low 
$(<1 \mathrm{~mm})$, suggesting that wrong assignments may "average out" because they occur equally in all directions. In an artificial setting, however, we showed that depending on the geometry of the imaged object, this compensation is not always possible, thus causing a large TRE.

In this study, the proposed variants for defining the covariance matrices as input for the anisotropic ICP yielded similar results. This can be attributed to the fact that we used the same reflectivity for all vertices and hence, similar standard deviations in each pixel. Furthermore, it should be noted that the training and testing conditions for ANISOTROPIC_SIM were similar. Future studies should account for these aspects.

In conclusion, the proposed registration method is well suited for dealing with anisotropic noise in the context of ToF range data registration, yielding a low TRE and a high percentage of correct vertex correspondences. Future work includes (1) assessment of convergence speed and accuracy with respect to the initial pose, (2) comparison with other variants of the ICP as well as with other methods for point registration in the presence of anisotropic noise, (3) evaluation on in-vitro and in-vivo data, and (4) validation of the extented ToF simulator.

\section{References}

1. Balachandran, R., Fitzpatrick, J.M.: Iterative solution for rigid-body point-based registration with anisotropic weighting. In: SPIE Medical Imaging, vol. 7261, p. 72613D (2009)

2. Kolb, A., et al.: Time-of-Flight sensors in computer graphics. In: Eurographics State of the Art Reports, pp. 119-134 (2009)

3. Besl, P.J., et al.: A method for registration of 3-d shapes. IEEE T. Pattern Anal. 14, 239-256 (1992)

4. Maier-Hein, L., et al.: Iterative closest point algorithm in the presence of anisotropic noise. In: Bildverarbeitung für die Medizin (BVM), pp. 231-235. Springer, Heidelberg (2010)

5. Danilchenko, A., Fitzpatrick, J.M.: General approach to error prediction in point registration. In: SPIE Medical Imaging, vol. 7625, p. 76250 F (2010)

6. Schmidt, M., Jähne, B.: A physical model of Time-of-Flight 3D imaging systems, including suppression of ambient light. In: Kolb, A., Koch, R. (eds.) Dyn3D 2009. LNCS, vol. 5742, pp. 1-15. Springer, Heidelberg (2009)

7. Erz, M., Jähne, B.: Radiometric and spectrometric calibrations, and distance noise measurement of ToF cameras. In: Kolb, A., Koch, R. (eds.) Dyn3D 2009. LNCS, vol. 5742, pp. 28-41. Springer, Heidelberg (2009) 